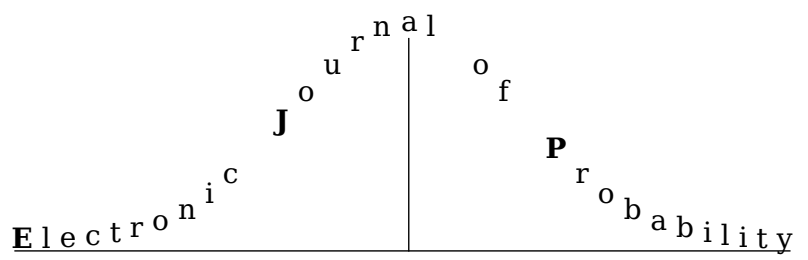

Electron. J. Probab. 26 (2021), article no. 46, 1-21.

ISSN: 1083-6489 https://doi.org/10.1214/21-EJP613

\title{
A localization theorem for the planar Coulomb gas in an external field
}

\author{
Yacin Ameur*
}

\begin{abstract}
We examine a two-dimensional Coulomb gas consisting of $n$ identical repelling point charges at an arbitrary inverse temperature $\beta$, subjected to a suitable external field.

We prove that the gas is effectively localized to a small neighbourhood of the droplet - the support of the equilibrium measure determined by the external field. More precisely, we prove that the distance between the droplet and the vacuum is with very high probability at most proportional to
\end{abstract}

$$
\sqrt{\frac{\log n}{\beta n}} .
$$

This order of magnitude is known to be "tight" when $\beta=1$ and the external field is radially symmetric.

In addition, we prove estimates for the one-point function in a neighbourhood of the droplet, proving in particular a fast uniform decay as one moves beyond a distance roughly of the order $\sqrt{\frac{\log n}{\beta n}}$ from the droplet.

Keywords: Coulomb gas; external potential; droplet; localization. MSC2020 subject classifications: $60 \mathrm{~K} 35$.

Submitted to EJP on September 8, 2019, final version accepted on March 29, 2021.

\section{Introduction and main results}

The planar Coulomb gas is a random configuration consisting of many (but finitely many) identical repelling point charges $\left\{\zeta_{i}\right\}_{1}^{n}$ in $\mathbb{C}$.

To keep the system from dispersing to infinity we assume the presence of an external field $n Q$ where $Q$ is a suitable extended real-valued function defined on $\mathbb{C}$, large near infinity in the sense that

$$
\liminf _{\zeta \rightarrow \infty} \frac{Q(\zeta)}{2 \log |\zeta|}>1 .
$$

${ }^{*}$ Lund University, Sweden. E-mail: yacin . ameur@math. lu.se 
The function $Q$, which is called an external potential, is fairly general but not quite arbitrary; precise assumptions are given below.

To a planar configuration $\left\{\zeta_{j}\right\}_{1}^{n}$ we associate the Hamiltonian (or energy)

$$
H_{n}=\sum_{j \neq k}^{n} \log \frac{1}{\left|\zeta_{j}-\zeta_{k}\right|}+n \sum_{j=1}^{n} Q\left(\zeta_{j}\right) .
$$

The statistical model is completed by fixing an inverse temperature $\beta=1 / k_{B} T$ and considering $\left\{\zeta_{j}\right\}_{1}^{n}$ as a random sample with respect to the Gibbs measure

$$
d \mathbf{P}_{n}^{\beta}=\frac{1}{Z_{n}^{\beta}} e^{-\beta H_{n}} d A_{n} .
$$

Here and throughout we write $d A$ for the Lebesgue measure in $\mathbb{C}$ divided by $\pi$ and $d A_{n}\left(\zeta_{1}, \ldots, \zeta_{n}\right)=d A\left(\zeta_{1}\right) \cdots d A\left(\zeta_{n}\right)$ for the corresponding product measure, where the constant $Z_{n}^{\beta}$ in (1.3) is the usual partition function,

$$
Z_{n}^{\beta}=\int_{\mathbb{C}^{n}} e^{-\beta H_{n}} d A_{n}
$$

We shall now gradually become explicit about our precise assumptions, introducing simultaneously our basic objects of study.

(i) The external potential $Q$ is assumed to be a fixed lower semi-continuous function on $\mathbb{C}$ with values in $\mathbb{R} \cup\{+\infty\}$ such that the set $\Sigma:=\{Q<+\infty\}$ has non-empty interior Int $\Sigma$. We also suppose that $Q$ is $C^{2}$-smooth in Int $\Sigma$, and that $Q$ obeys the growth condition (1.1).

We next define the logarithmic $Q$-energy of a finite, compactly supported Borel measure $\mu$ on $\mathbb{C}$ by

$$
I_{Q}[\mu]=\iint_{\mathbb{C}^{2}} \log \frac{1}{|\zeta-\eta|} d \mu(\zeta) d \mu(\eta)+\mu(Q),
$$

where " $\mu(Q)$ " is shorthand for $\int_{\mathbb{C}} Q d \mu$.

It is shown in [25] that there is a unique equilibrium measure $\sigma$ of total mass 1 , which minimizes $I_{Q}[\mu]$ over all compactly supported Borel probability measures $\mu$. The support of $\sigma$, which we denote by the symbol

$$
S=S[Q]:=\operatorname{supp} \sigma
$$

is called the droplet in external potential $Q$; we stress that this is a compact set. It is convenient to make the following assumption.

(ii) We assume that the droplet $S=S[Q]$ is contained in Int $\Sigma$ where $\Sigma=\{Q<+\infty\}$.

By (i) and (ii) it follows that $Q$ is smooth in a neighbourhood of $S$. It is well-known (see [25]) that in this circumstance, the equilibrium measure $\sigma$ is absolutely continuous with respect to $d A$ and takes the form

$$
d \sigma=\Delta Q \cdot \mathbf{1}_{S} d A .
$$

Here and in what follows we normalize the Laplacian by

$$
\Delta Q:=\partial \bar{\partial} Q=\frac{1}{4}\left(Q_{x x}+Q_{y y}\right), \quad(z=x+i y) .
$$

Observe that since $\sigma$ is a probability measure, $Q$ is necessarily subharmonic on the support $S$.

We now list additional conditions which we always assume to be satisfied in the sequel. 
(iii) The potential $Q$ is strictly subharmonic in a neighbourhood of the boundary $\partial S$.

(iv) The boundary $\partial S$ has finitely many components.

(v) Each component of $\partial S$ is an everywhere $C^{1}$-smooth Jordan curve.

(vi) $S^{*}=S$ where $S^{*}$ is the coincidence set for the obstacle problem, given in Section 2 .

Some of these conditions are assumed merely for convenience and may be relaxed. The practically minded reader may note that the class of potentials which are realanalytic where finite typically works well, and sufficiently small smooth perturbations of such potentials are also manageable. A detailed discussion of this situation is given in Subsection 5.1.

We will denote the Euclidean distance between a point $\zeta \in \mathbb{C}$ and the compact set $S$ by the symbol

$$
\delta(\zeta)=\operatorname{dist}(\zeta, S):=\min \{|\zeta-\eta| ; \eta \in S\}
$$

Let $\left\{\zeta_{j}\right\}_{1}^{n}$ be a random sample from (1.3). If $W$ is a measurable subset of $\mathbb{C}$ we will denote by $\#\left(W \cap\left\{\zeta_{j}\right\}_{1}^{n}\right)$ the number of indices $j$ such that $\zeta_{j} \in W$. By definition, the one-point function of $\left\{\zeta_{j}\right\}_{1}^{n}$ is

$$
\mathbf{R}_{n}^{\beta}(\zeta)=\lim _{\varepsilon \rightarrow 0} \frac{\mathbf{E}_{n}^{\beta}\left[\#\left(D(\zeta ; \varepsilon) \cap\left\{\zeta_{j}\right\}_{1}^{n}\right)\right]}{\varepsilon^{2}}
$$

where $D(\zeta ; \varepsilon)$ is the open disc $\{\eta ;|\zeta-\eta|<\varepsilon\}$.

We next define a positive constant $c_{0}=c_{0}[Q]$ by

$$
c_{0}:=\min \{\Delta Q(\eta) ; \eta \in \partial S\}
$$

Given these preliminaries, we have the following theorem.

Theorem 1. Let $Q$ be a potential satisfying assumptions (i)-(vi). There exists an open neighbourhood $\Omega$ of $S$ and constant $C>1$ such that for all $n \geq 1$

$$
\mathbf{R}_{n}^{\beta}(\zeta) \leq C^{\beta} n^{2} e^{-c \beta n \cdot \delta(\zeta)^{2}}, \quad \zeta \in \Omega .
$$

Here $c$ can be taken to be any positive constant with $c<c_{0}$.

The exterior decay given in (1.7) comes close to known exact results for $\beta=1$ as well as projected (or "physical") results for arbitrary $\beta$ in [12].

We note that the uniform bound $\mathbf{R}_{n}^{\beta}(\zeta) \leq C^{\beta} n^{2}$ of Theorem 1 can be improved in the determinantal case $\beta=1$ to $\mathbf{R}_{n}^{1} \leq C n$ (e.g. [5, Section 3]). We are not aware of similar uniform bounds for $\beta \neq 1$.

Associated to a random sample $\left\{\zeta_{j}\right\}_{1}^{n}$ we now define the number

$$
D_{n}:=\max _{1 \leq j \leq n}\left\{\delta\left(\zeta_{j}\right)\right\}
$$

We regard $D_{n}$ as a random variable with respect to the Gibbs measure, which thus represents the distance from the droplet to the vacuum.

Theorem 2. Assume that the external potential $Q$ satisfies assumptions (i)-(vi).

Let $\beta=\beta_{n}$ be a possibly $n$-dependent inverse temperature which is not too small in the sense that

$$
\lim _{n \rightarrow \infty} \frac{\beta_{n} n}{\log n}=\infty .
$$


Then there exists a sequence $\mu=\mu_{n}$ of positive numbers with

$$
\mu_{n} \lesssim \log \log n+\beta_{n}
$$

and constants $c>0$ and $a>0$, such that for each real $t$ satisfying

$$
t \leq a \beta_{n} n
$$

we have the estimate

$$
\mathbf{P}_{n}^{\beta}\left(\left\{D_{n}>\sqrt{\frac{\log n+\mu_{n}+t}{c \beta_{n} n}}\right\}\right) \leq e^{-2 t} .
$$

Here $c$ and a depend only on $Q ; c$ can be taken as any positive number with $c<c_{0}$, where $c_{0}$ is given in (1.6).

Remark 1.1. The meaning of the notation $a_{n} \lesssim b_{n}$ is that there exists a number $n_{0}$ and a constant $C$ such that $a_{n} \leq C b_{n}$ for all $n \geq n_{0}$. (The number $n_{0}$ may depend on the sequence $\left(\beta_{n}\right)$ and $Q$, while $C$ depends only on $n_{0}$ and $Q$.) The symbol $a_{n} \asymp b_{n}$ means $a_{n} \lesssim b_{n}$ and $b_{n} \lesssim a_{n}$.

Recall from [20] that (for any fixed $\beta>0$ ) the system $\left\{\zeta_{j}\right\}_{1}^{n}$ tends to follow the equilibrium measure $\sigma$ in the sense that

$$
\lim _{n \rightarrow \infty} \frac{1}{n} \mathbf{E}_{n}^{\beta}\left(f\left(\zeta_{1}\right)+\cdots+f\left(\zeta_{n}\right)\right) \rightarrow \sigma(f)
$$

for each bounded continuous function $f$. This implies, in a loose sense, that that the particles are likely to stay in the immediate vicinity of the droplet. It could be said that Theorem 2 gives more detailed information about exactly how "localized" the gas is about the droplet.

To illustrate this point, we may observe that if we fix a $\beta>0$ and choose $t=t_{n}$ so that $t_{n} \rightarrow \infty$ and $t_{n} / \log n \rightarrow 0$ as $n \rightarrow \infty$, then (1.11) implies

$$
A>\sqrt{\frac{1}{c_{0}}} \quad \text { implies } \quad \lim _{n \rightarrow \infty} \mathbf{P}_{n}^{\beta}\left(\left\{D_{n}>A \sqrt{\frac{\log n}{\beta n}}\right\}\right)=0 .
$$

Hence if $A$ satisfies the premise in (1.13), then the gas is effectively localized to the set of $\zeta$ with

$$
\delta(\zeta)<A \sqrt{\frac{\log n}{\beta n}} .
$$

The estimate (1.14) might be compared with earlier results on the distribution of the spectral radius of certain types of normal random matrices, due to Rider [23] for the Ginibre ensemble, cf. [16] for more general ensembles corresponding to radially symmetric potentials $Q$.

Indeed, as is well-known, we can interpret the Coulomb gas $\left\{\zeta_{j}\right\}_{j=1}^{n}$ in external potential $Q$ at inverse temperature $\beta=1$ as eigenvalues of normal random matrices. (See $[17,19]$ for details.) Let us temporarily assume that $Q$ is radially symmetric and that the droplet is the disc centered at 0 of radius $R$.

A normal matrix with eigenvalues $\left\{\zeta_{j}\right\}_{1}^{n}$ has its spectral radius equal to $\max _{1 \leq j \leq n}\left|\zeta_{j}\right|$, and if $D_{n}>0$ then clearly

$$
R+D_{n}=\max _{1 \leq j \leq n}\left|\zeta_{j}\right|
$$

The identity (1.15) may of course fail if $D_{n}=0$, but since $\mathbf{P}_{n}^{1}\left(\left\{D_{n}=0\right\}\right) \rightarrow 0$ rapidly as $n \rightarrow \infty$ by estimates in [16, 23], we may regard (1.15) as "essentially" an identity. 
We shall show in Subsection 5.2 that the estimate (1.14) comes close to earlier results on spectral radii in $[23,16]$, in the sense that the order of magnitude of our obtained localization is comparable with what is obtained in those papers.

While our main focus is on the case when $t=t_{n}$ increases slowly to infinity in the sense that $t_{n} / \beta n$ is "small", it is also relevant to note the following theorem, concerning the case when $t_{n} / \beta n$ is "large".

Theorem 3. Keeping the conditions in Theorem 2, there exists a number $r_{0}>0$ such that for each $r \geq r_{0}$ there is a number $k(r)>0$ such that

$$
\mathbf{P}_{n}^{\beta_{n}}\left(\left\{D_{n}>r\right\}\right) \lesssim \exp \left(-k(r) \cdot \beta_{n} n\right) .
$$

Indeed, we may take $k(r)$ proportional to $\min \left\{Q^{\mathrm{eff}}(\zeta) ; \delta(\zeta) \geq r\right\}$ where $Q^{\mathrm{eff}}$ is the effective potential defined in Section 2.

We remark that Theorem 3 is closely related to a result of Chafaï, Hardy, and Maïda, which holds in dimension $d \geq 2$. See [15, Theorem 1.12].

\section{Plan of this paper}

In Section 2 we give some background on potential theory and weighted polynomials. Theorem 1 is proven in Section 3 while Section 4 contains proofs of Theorem 2 and Theorem 3. In Section 5 we will state and prove generalized versions of the above theorems, and we also discuss some related earlier work in the area.

\section{Preparation}

In order to make this note as detailed and complete as possible, we shall now review some notions from the theories of obstacle problems and of weighted polynomials. We shall also discuss, in a suitably adapted form, some relevant background from [3].

As general sources for some statements taken for granted below, we refer to the book [25] and the paper [20].

We stress, once and for all, that in the following, the external potential $Q$ is assumed to be a fixed function satisfying assumptions (i)-(vi) above.

\subsection{The obstacle problem}

Let $\mathcal{F}_{Q}$ be the family of all subharmonic functions $f$ on $\mathbb{C}$ which are everywhere $\leq Q$ and which satisfy $f(\zeta) \leq 2 \log |\zeta|+O(1)$ as $\zeta \rightarrow \infty$.

We define a subharmonic function $\check{Q}$ on $\mathbb{C}$ by

$$
\check{Q}(\zeta)=\sup \left\{f(\zeta) ; f \in \mathcal{F}_{Q}\right\} .
$$

This is the obstacle function corresponding to the obstacle $Q$; it is well-known and easy to check that $\check{Q}$ satisfies $\check{Q} \leq Q$ and $\check{Q}(\zeta)=2 \log |\zeta|+O(1)$ as $\zeta \rightarrow \infty$.

Borrowing notation from [9], we define the effective potential

$$
Q^{\mathrm{eff}}:=Q-\check{Q},
$$

and note that $Q^{\text {eff }} \geq 0$ on $\mathbb{C}$. By the coincidence set for the obstacle problem we shall mean the compact set

$$
S^{*}:=\left\{Q^{\mathrm{eff}}=0\right\} .
$$

It is well-known (cf. [20]) that $\check{Q}$ is $C^{1,1}$-smooth on $\mathbb{C}$ and harmonic in the complement $\left(S^{*}\right)^{c}$. ("C $C^{1,1}$-smooth" means that the gradient is Lipschitz continuous.) Moreover, $\check{Q}$ is related to the equilibrium measure $\sigma$ by

$$
\check{Q}(\zeta)=-2 U^{\sigma}(\zeta)+\gamma
$$


where $\gamma$ is a suitable (Robin's) constant and

$$
U^{\sigma}(\zeta):=\int_{\mathbb{C}} \log \frac{1}{|\zeta-\eta|} d \sigma(\eta)
$$

is the logarithmic potential of $\sigma$.

Differentiating in the sense of distributions we have

$$
\Delta \check{Q}=\Delta Q \cdot \mathbf{1}_{S^{*}}=-2 \Delta U_{\sigma}=\sigma .
$$

Hence, since $S$ is the support of $\sigma$, we have the inclusion

$$
S \subset S^{*}
$$

In general the difference set $S^{*} \backslash S$ may be non-empty, consisting then of "shallow points" in the parlance of [20]. However, our assumption (vi) says precisely that there are no shallow points, i.e., that $S^{*}=S$. Thus condition (vi) can be restated as that

$$
Q^{\mathrm{eff}}>0 \quad \text { on } \quad S^{c} \text {. }
$$

(Here and in the following, $S^{c}:=\mathbb{C} \backslash S$.)

We will have frequent use for the following simple lemma. A proof is included for completeness.

Lemma 2.1. There exists a number $a_{0}>0$ such that

$$
Q^{\mathrm{eff}}(\zeta) \geq 2 \min \left\{c \cdot \delta(\zeta)^{2}, a_{0}\right\} \quad \text { for all } \quad \zeta \in S^{c} .
$$

Here the constant $c$ is any positive number with $c<c_{0}$ (cf. (1.6)).

Proof. Fix a boundary point $p \in \partial S$ and let $N=N_{p}$ be the unit normal to $\partial S$ at $p$ pointing outwards from $S$. Let $V$ be a $C^{2}$-smooth (Whitney's) extension of $\left.Q^{\text {eff }}\right|_{S^{c}}$ to a neighbourhood of $\partial S$.

We shall write $\partial_{N} V(p)$ for the directional derivative in direction $N$ and $\partial_{T} V(p)$ for the derivative in the (positively oriented) tangential direction to $\partial S$.

By the $C^{1,1}$-smoothness of $Q^{\text {eff }}$ and the fact that $Q^{\text {eff }}=0$ on $S$ we have $V(p)=$ $\partial_{N} V(p)=0$. Moreover, $\left(\partial_{N}^{2}+\partial_{T}^{2}\right) V(p)=4 \Delta Q(p)>0$ and $\partial_{T}^{2} V(p)=0$.

For small $\delta>0$ we hence obtain by Taylor's formula that

$$
Q^{\mathrm{eff}}(p+\delta N)=V(p+\delta N)=2 \Delta Q(p) \delta^{2}+o\left(\delta^{2}\right), \quad(\delta \rightarrow 0+) .
$$

Moreover, there is $\delta_{0}>0$ such that $Q^{\text {eff }}(\zeta) \geq 2 c \delta(\zeta)^{2}$ when $\delta(\zeta) \leq \delta_{0}$.

Finally, by the lower semi-continuity of $Q^{\text {eff }}$ and the assumptions (1.1), (2.1) we conclude that $Q^{\text {eff }}$ attains a strictly positive minimum over the set $\left\{\delta(\zeta) \geq \delta_{0}\right\}$. The lemma follows if we denote this minimum value by " $2 a_{0}$ ".

We next note the following lemma - a simple consequence of our growth assumption on the external potential $Q$.

Lemma 2.2. $\int_{\mathbb{C}} Q e^{-Q} d A<\infty$ where $Q e^{-Q}:=0$ on $\{Q=+\infty\}$.

Proof. Let $\alpha$ be a number in the range $1<\alpha<\liminf _{\zeta \rightarrow \infty} Q(\zeta) / \log |\zeta|^{2}$, cf. (1.1). Fix a number $\theta<1$ such that $\theta \alpha>1$ and let $x_{\theta}$ be a real number such that $x e^{-x} \leq e^{-\theta x}$ when $x \geq x_{\theta}$. Choosing $x_{\theta}$ somewhat larger if necessary we can also assume that $Q(\zeta) \geq \alpha \log |\zeta|^{2}$ when $|\zeta| \geq x_{\theta}$. Then $Q(\zeta) e^{-Q(\zeta)} \leq|\zeta|^{-2 \alpha \theta}$ when $|\zeta| \geq x_{\theta}$, proving the lemma. 


\subsection{Weighted polynomials}

Let $\mathscr{W}_{n}$ denote the subspace of $L^{2}=L^{2}(\mathbb{C}, d A)$ consisting of elements (weighted polynomials)

$$
f=q \cdot e^{-n Q / 2}
$$

where $q$ is a holomorphic polynomial of degree at most $n-1$.

The following well-known lemma is sometimes known as the "maximum principle of weighted potential theory". We outline a proof for convenience.

Lemma 2.3. If $f \in \mathscr{W}_{n}$ and $\zeta \in \mathbb{C}$ then

$$
|f(\zeta)| \leq\|f\|_{L^{\infty}(S)} \cdot e^{-n Q^{\mathrm{eff}}(\zeta) / 2}
$$

Proof. We may assume that $\|f\|_{L^{\infty}(S)}=1$ where $f=q \cdot e^{-n Q / 2}$. Since

$$
\frac{1}{n} \log |q(\zeta)|^{2}=\frac{1}{n} \log |f(\zeta)|^{2}+Q(\zeta)
$$

we see that the function $u:=\frac{1}{n} \log |q|^{2}$, which is subharmonic on $\mathbb{C}$, satisfies $u \leq Q$ on $S$, and furthermore $u(\zeta) \leq \log |\zeta|^{2}+O(1)$ as $\zeta \rightarrow \infty$.

A suitable version of the maximum principle now shows that $u \leq \check{Q}$ on $\mathbb{C}$, thus finishing the proof of the lemma.

We now fix, once and for all, an open neighbourhood $V$ of the droplet $S$, which is small enough so that $\Delta Q$ is continuous and strictly positive in a neighbourhood $V_{1}$ of the closure $\bar{V}$. This is possible by assumption (iii).

Next fix a number $s$ with

$$
s>\max \{\Delta Q(\zeta) ; \zeta \in \bar{V}\}
$$

Lemma 2.4. There exists $n_{0}>0$ such that if $n \geq n_{0}$ then for each $f \in \mathscr{W}_{n}$ and each $\zeta_{0} \in \bar{V}$ we have the pointwise- $L^{2 \beta}$ estimate

$$
\left|f\left(\zeta_{0}\right)\right|^{2 \beta} \leq n e^{s \beta} \int_{D\left(\zeta_{0} ; 1 / \sqrt{n}\right)}|f|^{2 \beta} d A
$$

Proof. Consider the function

$$
F(\zeta)=|f(\zeta)|^{2 \beta} e^{s n \beta|\zeta|^{2}}
$$

Writing $f=q \cdot e^{-n Q / 2}$ we have

$$
\Delta \log F(\zeta) \geq-\beta n \Delta Q(\zeta)+s \beta n \geq 0
$$

for all $\zeta \in V_{1}$. We infer that $F$ is logarithmically subharmonic in $V_{1}$, and in particular it is subharmonic there.

Applying the sub mean-value inequality, we obtain (for $n$ large enough that $1 / \sqrt{n} \leq$ $\left.\operatorname{dist}\left(V, V_{1}^{c}\right)\right)$ the estimate

$$
F\left(\zeta_{0}\right) \leq n \int_{D\left(\zeta_{0} ; 1 / \sqrt{n}\right)} F d A \leq n e^{s \beta} \int_{D\left(\zeta_{0} ; 1 / \sqrt{n}\right)}|f|^{2 \beta} d A
$$

The proof is complete. 


\subsection{Random variables}

Let $\left\{\zeta_{j}\right\}_{1}^{n}$ be a random sample with respect to the Gibbs measure (1.3).

Consider now, for a fixed $j$ with $j \in\{1, \ldots, n\}$ the random weighted Lagrange polynomial

$$
\ell_{j}(\zeta)=\left(\prod_{i \neq j}\left(\zeta-\zeta_{i}\right) / \prod_{i \neq j}\left(\zeta_{j}-\zeta_{i}\right)\right) \cdot e^{-n\left(Q(\zeta)-Q\left(\zeta_{j}\right)\right) / 2} .
$$

These weighted polynomials were used in [3] to study the separation of random configurations. We shall here use similar techniques to examine the localization of the gas.

Towards this end, let us fix a measurable subset $W \subset \mathbb{C}$ and write $Y_{j}=Y_{j, W}$ for the random variable

$$
Y_{j, W}:=\int_{W}\left|\ell_{j}(\zeta)\right|^{2 \beta} d A(\zeta)
$$

\subsection{Exact identities}

The following lemma is a slight generalization of [3, Lemma 2]; it will play a key rôle in what follows.

Lemma 2.5. Let $U \subset \mathbb{C}$ be a measurable subset, of $d A$-measure $|U|$. Then

$$
\mathbf{E}_{n}^{\beta}\left[\mathbf{1}_{U}\left(\zeta_{j}\right) \cdot Y_{j, W}\right]=|U| \cdot p_{n}^{\beta}(W), \quad(j=1, \ldots, n),
$$

where

$$
p_{n}^{\beta}(W):=\mathbf{P}_{n}^{\beta}\left(\left\{\zeta_{j} \in W\right\}\right)=\mathbf{P}_{n}^{\beta}\left(\left\{\zeta_{1} \in W\right\}\right) .
$$

Proof. We start with the basic identity

$$
\left|\ell_{j}(\zeta)\right|^{2 \beta} e^{-\beta H_{n}\left(\zeta_{1}, \ldots, \zeta_{j}, \ldots, \zeta_{n}\right)}=e^{-\beta H_{n}\left(\zeta_{1}, \ldots, \zeta_{,}, \ldots, \zeta_{n}\right)} .
$$

By Fubini's theorem, integrating first in $\zeta_{j}$, we get

$$
\begin{aligned}
& \int_{W} d A(\zeta) \mathbf{E}_{n}^{\beta}\left[\left|\ell_{j}(\zeta)\right|^{2 \beta} \cdot \mathbf{1}_{U}\left(\zeta_{j}\right)\right] \\
& \quad=\int_{U} d A\left(\zeta_{j}\right) \int_{\zeta \in W, \zeta_{k} \in \mathbb{C},(k \neq j)} d \mathbf{P}_{n}^{\beta}\left(\zeta_{1}, \ldots, \zeta, \ldots, \zeta_{n}\right)=|U| \cdot p_{n}^{\beta}(W) .
\end{aligned}
$$

The proof is complete.

The argument above may be iterated. To illuminate the principle, we start by considering the case of two different indices $j$ and $k, 1 \leq j<k \leq n$.

Lemma 2.6. If $j<k$ and $U_{1}, U_{2}, W_{1}, W_{2}$ are any measurable subsets of $\mathbb{C}$ then

$$
\mathbf{E}_{n}^{\beta}\left[\mathbf{1}_{U_{1}}\left(\zeta_{j}\right) \cdot Y_{j, W_{1}} \cdot \mathbf{1}_{U_{2}}\left(\zeta_{k}\right) \cdot Y_{k, W_{2}}\right]=\left|U_{1}\right|\left|U_{2}\right| \cdot p_{n, 2}^{\beta}\left(W_{1}, W_{2}\right),
$$

where

$$
p_{n, 2}^{\beta}\left(W_{1}, W_{2}\right):=\mathbf{P}_{n}^{\beta}\left(\left\{\zeta_{1} \in W_{1}, \zeta_{2} \in W_{2}\right\}\right) .
$$

Proof. Note that

$$
\left|\ell_{j}(\zeta)\right|^{2 \beta}\left|\ell_{k}(\eta)\right|^{2 \beta} e^{-\beta H_{n}\left(\zeta_{1}, \ldots, \zeta_{j}, \ldots, \zeta_{k}, \ldots, \zeta_{n}\right)}=e^{-\beta H_{n}\left(\zeta_{1}, \ldots, \zeta, \ldots, \eta, \ldots, \zeta_{n}\right)} .
$$

with $\zeta$ and $\eta$ in positions $j$ and $k$, respectively. The lemma follows by using Fubini's theorem as in the preceding proof. 
More generally, if $\left\{j_{1}, \ldots, j_{m}\right\}$ is any subset of $\{1, \ldots, n\}$ with $j_{1}<j_{2}<\cdots<j_{m}$ then with a self-explanatory notation

$$
\begin{aligned}
\mathbf{E}_{n}^{\beta}\left[\mathbf{1}_{U_{1}}\left(\zeta_{j_{1}}\right) \cdot Y_{j_{1}, W_{1}} \cdot \mathbf{1}_{U_{2}}\left(\zeta_{j_{2}}\right) \cdot Y_{j_{2}, W_{2}} \cdots \mathbf{1}_{U_{m}}\left(\zeta_{j_{m}}\right) \cdot Y_{j_{m}, W_{m}}\right] \\
=\left|U_{1}\right|\left|U_{2}\right| \cdots\left|U_{m}\right| \cdot p_{n, m}^{\beta}\left(W_{1}, \ldots W_{m}\right) .
\end{aligned}
$$

The proof of this formula is straightforward and is omitted.

In the following we will consider the case when all the sets $W_{j}$ coincide with a set $W$, in which case we will use the abbreviation

$$
p_{n, m}^{\beta}(W):=p_{n, m}^{\beta}(W, W, \ldots, W) .
$$

( $W$ " occurs $m$ times in the right hand side.)

\section{Proof of Theorem 1}

To begin, we fix a positive number $c<c_{0}$ and a small enough open neighbourhood $\Omega$ of $S$ such that $c \delta(\zeta)^{2}<a_{0}$ for each $\zeta \in \Omega$. (Here $c_{0}$ is fixed as in (1.6) and $a_{0}$ is the constant from Lemma 2.1.) We assume also that $\Omega$ is small enough that Lemma 2.4 applies with $V=\Omega$.

Now fix a point $\zeta_{0} \in \Omega$ and a number $\varepsilon>0$ which is small enough that the neighbourhood $W=D\left(\zeta_{0} ; \varepsilon\right)$ is contained in $\Omega$.

Next define a non-negative number $\delta$ by

$$
\delta:=\operatorname{dist}(S, W)=\inf \{|\zeta-\eta| ; \zeta \in S, \eta \in W\} .
$$

Thus $\delta \rightarrow \delta\left(\zeta_{0}\right)$ as $\varepsilon \rightarrow 0$.

For $1 \leq j \leq n$ we consider the random variables

$$
\begin{aligned}
& Y_{j}=\int_{W}\left|\ell_{j}\right|^{2 \beta} d A, \\
& Z_{j}=\int_{\mathbb{C}}\left|\ell_{j}\right|^{2 \beta} d A .
\end{aligned}
$$

By Lemma 2.4 we have the global estimate

$$
\left|\ell_{j}(\zeta)\right|^{2 \beta} \leq n e^{s \beta} \int_{D(\zeta, 1 / \sqrt{n})}\left|\ell_{j}\right|^{2 \beta} \leq n e^{s \beta} Z_{j}, \quad \zeta \in \Omega .
$$

In particular this holds for all $\zeta \in S$, so since $\ell_{j} \in \mathscr{W}_{n}$ we obtain from lemmas 2.3 and 2.1 that

$$
\left|\ell_{j}(\zeta)\right|^{2 \beta} \leq n e^{s \beta} e^{-c n \beta \delta^{2}} Z_{j}, \quad \zeta \in W .
$$

Integrating the latter inequality over $W$ using that $|W|=\varepsilon^{2}$, we find that

$$
Y_{j} \leq n e^{s \beta} e^{-c n \beta \delta^{2}} \varepsilon^{2} Z_{j} .
$$

Now fix a measurable subset $U$ of positive, finite measure and recall from Lemma 2.5 that

$$
\begin{aligned}
\mathbf{E}_{n}^{\beta}\left[\mathbf{1}_{U}\left(\zeta_{j}\right) \cdot Y_{j}\right] & =|U| \cdot p_{n}^{\beta}(W), \\
\mathbf{E}_{n}^{\beta}\left[\mathbf{1}_{U}\left(\zeta_{j}\right) \cdot Z_{j}\right] & =|U| \cdot p_{n}^{\beta}(\mathbb{C})=|U| .
\end{aligned}
$$

If we multiply through in (3.1) by $\mathbf{1}_{U}\left(\zeta_{j}\right)$ and then take expectations, we obtain the inequality

$$
p_{n}^{\beta}(W) \leq n e^{s \beta} e^{-c n \beta \delta^{2}} \varepsilon^{2} .
$$


Next define for $j \neq k$

$$
\begin{aligned}
Y_{j, k} & =\int_{W^{2}}\left|\ell_{j}(\zeta) \ell_{k}(\eta)\right|^{2 \beta} d A_{2}(\zeta, \eta), \\
Z_{j, k} & =\int_{\mathbb{C}^{2}}\left|\ell_{j}(\zeta) \ell_{k}(\eta)\right|^{2 \beta} d A_{2}(\zeta, \eta) .
\end{aligned}
$$

Applying the argument above, we find first that

$$
\left|\ell_{j}(\zeta) \ell_{k}(\eta)\right|^{2 \beta} \leq\left(n e^{s \beta} e^{-c n \beta \delta^{2}}\right)^{2} Z_{j, k}, \quad \zeta, \eta \in W
$$

and then, by integrating over $W^{2}$,

$$
Y_{j, k} \leq\left(n e^{s \beta} e^{-c n \beta \delta^{2}}\right)^{2} Z_{j, k} \varepsilon^{4}
$$

Taking expectations in this inequality and using Lemma 2.6 it now follows that

$$
p_{n, 2}^{\beta}(W) \leq\left(n e^{s \beta} e^{-c n \beta \delta^{2}}\right)^{2} p_{n, 2}^{\beta}(\mathbb{C}) \varepsilon^{4}=\left(n e^{s \beta} e^{-c n \beta \delta^{2}}\right)^{2} \varepsilon^{4} .
$$

By the same token, using the identity (2.4), we obtain for each $k, 1 \leq k \leq n$ that

$$
p_{n, k}^{\beta}(W) \leq q^{k} p_{n, k}^{\beta}(\mathbb{C}) \varepsilon^{2 k}=q^{k} \varepsilon^{2 k} \quad \text { where } \quad q=n e^{s \beta} e^{-c n \beta \delta^{2}}
$$

From this, we infer that

$$
\begin{aligned}
& \mathbf{E}_{n}^{\beta}\left(\#\left(W \cap\left\{\zeta_{j}\right\}_{1}^{n}\right)\right)=\mathbf{P}_{n}^{\beta}\left(\left\{\#\left(W \cap\left\{\zeta_{j}\right\}_{1}^{n}\right)=1\right\}\right) \\
& \quad+2 \mathbf{P}_{n}^{\beta}\left(\left\{\#\left(W \cap\left\{\zeta_{j}\right\}_{1}^{n}\right)=2\right\}\right)+\cdots+n \mathbf{P}_{n}^{\beta}\left(\left\{\#\left(W \cap\left\{\zeta_{j}\right\}_{1}^{n}\right)=n\right\}\right) \\
& \quad \leq\left(\begin{array}{c}
n \\
1
\end{array}\right) q \varepsilon^{2}+2\left(\begin{array}{c}
n \\
2
\end{array}\right) q^{2} \varepsilon^{4}+\cdots+n\left(\begin{array}{c}
n \\
n
\end{array}\right) q^{n} \varepsilon^{2 n} .
\end{aligned}
$$

We finally conclude that

$$
\mathbf{R}_{n}^{\beta}\left(\zeta_{0}\right)=\lim _{\varepsilon \rightarrow 0} \frac{\mathbf{E}_{n}^{\beta}\left(\#\left(W \cap\left\{\zeta_{j}\right\}_{1}^{n}\right)\right)}{\varepsilon^{2}} \leq n^{2} e^{s \beta} e^{-c n \beta \delta\left(\zeta_{0}\right)^{2}},
$$

as desired. Q.E.D.

\section{Proofs of Theorem 2 and Theorem 3}

We start by fixing a sequence $\left(U_{n}\right)_{n=1}^{\infty}$ of bounded open neighbourhoods of the droplet $S$, which is increasing and exhausts $\mathbb{C}$, viz.

$$
S \subset U_{1} \subset U_{2} \subset \cdots, \quad \bigcup_{n=1}^{\infty} U_{n}=\mathbb{C} .
$$

In the sequel we fix an unspecified integer $n_{0}$ which can be chosen larger as we go along, and we assume that $n \geq n_{0}$.

For definiteness, we will fix $U_{n}$ to be the disc of radius $\log n$ about the origin,

$$
U_{n}:=D\left(0 ; R_{n}\right), \quad R_{n}:=\log n, \quad\left(n \geq n_{0}\right)
$$

Now fix $j, 1 \leq j \leq n$, and put

$$
X_{j}=\int_{\mathbb{C}}\left|\ell_{j}\right|^{2 \beta} d A
$$


By Lemma 2.5 we know that

$$
\mathbf{E}_{n}^{\beta}\left[\mathbf{1}_{U_{n}}\left(\zeta_{j}\right) \cdot X_{j}\right]=\left|U_{n}\right|=R_{n}^{2} .
$$

Now introduce the two events

$$
A_{j}:=\left\{\zeta_{j} \in U_{n}\right\}, \quad B_{j}:=\left\{X_{j} \leq \lambda\right\},
$$

where $\lambda>0$ is a parameter.

By Chebyshev's inequality and (4.2) we have the basic estimate

$$
\mathbf{P}_{n}^{\beta}\left(A_{j} \cap B_{j}^{c}\right) \leq \frac{R_{n}^{2}}{\lambda} .
$$

Passing to complements we conclude that

$$
\mathbf{P}_{n}^{\beta}\left(A_{j}^{c}\right)+\mathbf{P}_{n}^{\beta}\left(B_{j}\right) \geq 1-\frac{R_{n}^{2}}{\lambda} .
$$

We shall now prove that the probability $\mathbf{P}_{n}^{\beta}\left(A_{j}^{c}\right)$ is "negligible".

Lemma 4.1. There are constants $h_{*}>0$ and $n_{0}>0$ such that $n \geq n_{0}$ implies

$$
\mathbf{P}_{n}^{\beta}\left(A_{j}^{c}\right) \leq R_{n}^{-\beta h_{*} n}, \quad 1 \leq j \leq n .
$$

Proof. We will give a proof based on estimates for the partition function which can essentially be found in the union of the papers [20,21]. ([21] is written in the setting of a real log-gas, but the following argument is virtually the same in the complex case.)

We first note, due to the growth assumption (1.1) on $Q$, that there are numbers $h_{0}>0$ and $n_{0}>0$ such that for all $\zeta, \eta \in \mathbb{C}$ with $|\eta|>R_{n_{0}}$ we have

$$
\log \frac{1}{|\zeta-\eta|^{2}}+Q(\zeta)+Q(\eta)>h_{0} \log |\eta|
$$

(To see this, use the elementary inequality $|\zeta-\eta|^{2} \leq\left(1+|\zeta|^{2}\right)\left(1+|\eta|^{2}\right)$.)

Recalling the definition of the partition function $Z_{n}^{\beta}$ in (1.4), we now write

$$
\begin{aligned}
& \mathbf{P}_{n}^{\beta}\left(A_{j}^{c}\right)=\mathbf{P}_{n}^{\beta}\left(A_{n}^{c}\right)=\mathbf{P}_{n}^{\beta}\left(\left\{\zeta_{n} \in U_{n}^{c}\right\}\right)=\frac{Z_{n-1}^{\beta}}{Z_{n}^{\beta}} \int_{U_{n}^{c}} e^{-n \beta Q\left(\zeta_{n}\right)} d A\left(\zeta_{n}\right) \times \\
& \quad \times \int_{\mathbb{C}^{n-1}} \exp \left(-\beta \sum_{j=1}^{n-1}\left[\log \frac{1}{\left|\zeta_{j}-\zeta_{n}\right|^{2}}+Q\left(\zeta_{j}\right)\right]\right) d \mathbf{P}_{n-1}^{\beta}\left(\zeta_{1}, \ldots, \zeta_{n-1}\right) .
\end{aligned}
$$

Using (4.5) we now obtain

$$
\begin{aligned}
\mathbf{P}_{n}^{\beta}\left(A_{j}^{c}\right) & \leq \frac{Z_{n-1}^{\beta}}{Z_{n}^{\beta}} \int_{U_{n}^{c}} e^{-\beta Q\left(\zeta_{n}\right)} e^{-\beta(n-1) h_{0} \log \left|\zeta_{n}\right|} d A\left(\zeta_{n}\right) \\
& \leq \frac{Z_{n-1}^{\beta}}{Z_{n}^{\beta}} R_{n}^{-\beta h_{0}(n-1) / 2} \int_{U_{n}^{c}} e^{-\beta Q\left(\zeta_{n}\right)}\left|\zeta_{n}\right|^{-\beta h_{0}(n-1) / 2} d A\left(\zeta_{n}\right) .
\end{aligned}
$$

We conclude that there are constants $C$ and $h_{1}>0$ such that

$$
\mathbf{P}_{n}^{\beta}\left(A_{j}^{c}\right) \leq C \frac{Z_{n-1}^{\beta}}{Z_{n}^{\beta}} R_{n}^{-\beta h_{1} n} .
$$

We shall thus be done when we can prove an upper bound of the form

$$
\frac{Z_{n-1}^{\beta}}{Z_{n}^{\beta}} \leq C e^{\beta h_{2} n} .
$$


For fixed $\beta$ this is shown in Section 5 of [15] (see eq. (5.4)), and it also follows formally from a well-known large $n$ expansion of the partition function in [28]. As we want to ensure a certain uniformity in $\beta$, we shall here give an elementary proof based on the papers $[20,21]$.

We start with the identity

$$
\frac{Z_{n}^{\beta}}{Z_{n-1}^{\beta}}=\mathbf{E}_{n-1}^{\beta}\left[\int_{\mathbb{C}} \exp \left(\beta \sum_{j=1}^{n-1} \log \left|t-\zeta_{j}\right|^{2}-n \beta Q(t)\right) d A(t)\right] .
$$
is

Now write $I:=\int_{\mathbb{C}} e^{-Q}$ and use Jensen's inequality to conclude that the last expression

$$
\geq I \cdot \exp \left\{\mathbf{E}_{n-1}^{\beta}\left[\int_{\mathbb{C}}\left(\beta \sum_{j=1}^{n-1} \log \left|t-\zeta_{j}\right|^{2}-(\beta n-1) Q(t)\right) \frac{e^{-Q(t)}}{I} d A(t)\right]\right\} .
$$

We must estimate this expression from below. For this, we start by estimating the number

$$
\begin{aligned}
m_{n}: & =\frac{1}{n} \mathbf{E}_{n-1}^{\beta}\left[\int_{\mathbb{C}} \sum_{j=1}^{n-1} \log \left|t-\zeta_{j}\right|^{2} e^{-Q(t)} d A(t)\right] \\
& \geq \frac{1}{n} \mathbf{E}_{n-1}^{\beta}\left[\int_{\mathbb{C}} \sum_{j=1}^{n-1} \log _{-}\left|t-\zeta_{j}\right|^{2} e^{-Q(t)} d A(t)\right]
\end{aligned}
$$

where $\log _{-} x=\min \{\log x, 0\}$.

Now fix a number $\delta, 0<\delta<1$ so that $\int_{|t-\zeta|<\delta} \log |t-\zeta|^{2} e^{-Q(t)} d A(t)>-1$ for each $\zeta \in \mathbb{C}$. Then

$$
\begin{array}{r}
m_{n}>-1+\frac{1}{n} \mathbf{E}_{n-1}^{\beta}\left[\sum_{j=1}^{n-1} \int_{\left|t-\zeta_{j}\right| \geq \delta} \log _{-}\left|t-\zeta_{j}\right|^{2} e^{-Q(t)} d A(t)\right] \\
>-1+2 \log \delta+\frac{1}{n} \mathbf{E}_{n-1}^{\beta}\left[\int_{\mathbb{C}} \sum_{j=1}^{n-1} l_{\delta}\left(t-\zeta_{j}\right) e^{-Q(t)} d A(t)\right],
\end{array}
$$

where $l_{\delta}(\zeta):=2 \max \left\{\log _{-}|\zeta|, \log \delta\right\}$.

Now as $l_{\delta}$ is bounded and continuous on $\mathbb{C}$ we can apply the convergence in (1.12) to obtain

$$
\frac{1}{n} \mathbf{E}_{n-1}^{\beta}\left(l_{\delta}\left(t-\zeta_{1}\right)+\cdots+l_{\delta}\left(t-\zeta_{n-1}\right)\right) \rightarrow \int_{\mathbb{C}} l_{\delta}(t-\zeta) d \sigma(\zeta)
$$

for each $t \in \mathbb{C}$.

In fact, an examination of the proof of the convergence $(1.12)$ in $[20,21]$ holds uniformly in $t$ provided that $\beta=\beta_{n}$ satisfies $\beta_{n} n \rightarrow \infty$ as $n \rightarrow \infty$. For convenience of the reader, we have collected the relevant details in the appendix; see in particular Theorem A. 1 .

Integrating both sides of the limit (4.10) with respect to the measure $e^{-Q(t)} d A(t)$ we infer that the sequence $m_{n}$ is uniformly bounded from below when $\beta \gg \frac{\log n}{n}$, which is all that we need to know here.

In view of Lemma 2.2, we now obtain by (4.8) and (4.9) that a bound of the form (4.7) must hold.

The proof of the lemma is complete. 
Remark 4.2. If we slightly strengthen our assumptions on the potential, then the above uniform convergence in (4.10) also follows from a quantitative result in [15, Theorem 1.5]. (The function $l_{\delta}$ belongs to the bounded Lipschitz class used there.)

\subsection{Proof of Theorem 2}

Now fix an $n \geq n_{0}$ and recall that $B_{j}=\left\{X_{j} \leq \lambda\right\}$ where $\lambda>0$ is fixed. (See (4.3).)

It follows from Lemma 4.1 and the inequality (4.4) that

$$
\mathbf{P}_{n}^{\beta}\left(B_{j}\right) \geq 1-R_{n}^{2} \lambda^{-1}-R_{n}^{-\beta h_{*} n}, \quad j=1, \ldots, n .
$$

In order to be able to neglect the last term, we shall impose the following (mild) condition on the parameter $\lambda$,

$$
\lambda \lesssim R_{n}^{\beta h_{*} n / 2} .
$$

Under this assumption we have

$$
\mathbf{P}_{n}^{\beta}\left(B_{j}\right) \geq 1-K R_{n}^{2} \lambda^{-1}
$$

for some constant $K$. Hence if we write

$$
B:=\bigcap_{j=1}^{n} B_{j},
$$

then

$$
\mathbf{P}_{n}^{\beta}(B) \geq 1-\epsilon,
$$

where we have put

$$
\epsilon:=K n R_{n}^{2} \lambda^{-1} .
$$

In the sequel we assume that $\zeta$ belongs to the fixed neighbourhood $V$ of $S$. Pick a random sample $\left\{\zeta_{j}\right\}_{1}^{n}$.

By Lemma 2.4 we have that

$$
\left|\ell_{j}\right|^{2 \beta}(\zeta) \leq n e^{s \beta} X_{j}, \quad\left(X_{j}=\int\left|\ell_{j}\right|^{2 \beta}\right) .
$$

Thus assuming that $B_{j}$ has occurred, we obtain

$$
\left\|\ell_{j}\right\|_{L^{\infty}(S)}^{2 \beta} \leq n e^{s \beta} \lambda .
$$

We now apply Lemma 2.3 to conclude that

$$
\left|\ell_{j}(\zeta)\right|^{2 \beta} \leq n e^{s \beta} \lambda \cdot e^{-n \beta Q^{\text {eff }}(\zeta)}, \quad(\zeta \in \mathbb{C}) .
$$

In view of Lemma 2.1 we deduce that if $\zeta \in S^{c}$ then

$$
\left|\ell_{j}(\zeta)\right|^{2 \beta} \leq n e^{s \beta} \lambda \exp \left(-2 n \beta \min \left\{c \delta(\zeta)^{2}, a_{0}\right\}\right) .
$$

To proceed, we now impose the further restriction on $\lambda$ that

$$
\lambda \lesssim e^{n \beta a_{0}} .
$$

We also fix an arbitrary number $\alpha \in(0,1)$ and choose $n_{0}$ large enough that $n \geq n_{0}$ implies

$$
n e^{s \beta} \lambda e^{-2 n \beta a_{0}}<\alpha .
$$


This is possible by (4.16) since (1.9) implies that $n \beta a_{0}>\log n$ for large $n$.

For a fixed $n \geq n_{0}$ we now consider the set $M_{n} \subset S^{c}$ of points $\zeta$ such that

$$
n e^{s \beta} \lambda e^{-2 c \beta n \delta(\zeta)^{2}} \leq \alpha .
$$

Note that if $B_{j}$ has occurred then certainly $\zeta_{j} \notin M_{n}$, for otherwise (4.15) would imply

$$
\alpha \geq\left|\ell_{j}\left(\zeta_{j}\right)\right|^{2 \beta}=1>\alpha .
$$

Thus with probability at least $1-\epsilon$ the entire Coulomb gas is actually contained in the neighbourhood $M_{n}^{c}$ of $S$.

To finish the proof we observe that our restrictions on $\lambda$ are equivalent to that the parameter $\epsilon$ (cf. (4.13)) satisfy

$$
\epsilon \gtrsim n R_{n}^{2} e^{-n \beta a_{0}} \quad \text { and } \quad \epsilon \gtrsim n R_{n}^{2} e^{-\beta h_{*} n / 2} .
$$

For such $\epsilon$ we now write

$$
\lambda=\frac{n \nu_{n}}{\epsilon}, \quad \text { where } \quad \nu_{n}=K R_{n}^{2}+o(1) .
$$

The inequality (4.18) is then equivalent to that

$$
n^{2} e^{s \beta} \frac{\nu_{n}}{\epsilon} \leq \alpha e^{2 c \beta n \delta^{2}}, \quad(\delta=\delta(\zeta)),
$$

which transforms to

$$
\delta^{2} \geq \frac{1}{2 c \beta n}\left(\log \frac{1}{\alpha}+s \beta+2 \log n+\log \frac{\nu_{n}}{\epsilon}\right) .
$$

Thus if we define

$$
\delta_{n}=\sqrt{\frac{1}{2 c \beta n}\left(\log \frac{1}{\alpha}+s \beta+2 \log n+\log \frac{\nu_{n}}{\epsilon}\right)}
$$

then certainly

$$
M_{n} \subset\left\{\zeta \in \mathbb{C} ; \delta(\zeta) \geq \delta_{n}\right\} .
$$

so the Coulomb gas is with probability at least $1-\epsilon$ contained in the $\delta_{n}$-neighbourhood of $S$. In symbols, we have shown that

$$
\mathbf{P}_{n}^{\beta}\left(\left\{D_{n} \geq \delta_{n}\right\}\right) \leq \epsilon .
$$

If we write $\epsilon=e^{-t}$ and $\mu_{n}=\log \nu_{n}+\log (1 / \alpha)+s \beta$ this becomes

$$
\mathbf{P}_{n}^{\beta}\left(\left\{D_{n}>\sqrt{\frac{1}{c \beta n}\left(\log n+\mu_{n} / 2+t / 2\right)}\right\}\right) \leq e^{-t} .
$$

Now notice that $\log \nu_{n} \asymp \log \log n$ as $n \rightarrow \infty$, so $\mu_{n} \asymp \log \log n+\beta$.

We have shown (4.20) under the hypothesis (4.19). Since we have assumed that $\beta \cdot n \rightarrow \infty$ as $n \rightarrow \infty$, (4.19) surely holds if $t \leq a \beta n$ for a small enough $a>0$.

The proof of Theorem 2 is complete. Q.E.D.

\subsection{Proof of Theorem 3}

It is now easy to modify the above proof so as to also prove Theorem 3.

Fix a number $r_{0}$ with $c r_{0}^{2} \geq a_{0}$ and suppose that $\delta(\zeta) \geq r \geq r_{0}$. The estimate (4.15) then takes the form

$$
\left|\ell_{j}(\zeta)\right|^{2 \beta} \leq n e^{s \beta} \lambda e^{-2 n \beta k_{0}},
$$


where $k_{0}$ is some constant with $k_{0} \geq a_{0}$. A glance at the proof of Lemma 2.1 shows that we may take $2 k_{0}=\min \left\{Q^{\text {eff }}(\zeta) ; \delta(\zeta) \geq r\right\}$.

For a fixed $\alpha \in(0,1)$ we now choose $n_{0}$ large enough that $n \geq n_{0}$ implies

$$
n e^{s \beta} \lambda e^{-2 n \beta k_{0}} \leq \alpha .
$$

This can certainly be done if we assume that $\lambda \lesssim e^{n \beta k_{0}}$, in view of (1.9).

If the event $B_{j}=\left\{X_{j} \leq \lambda\right\}$ has occurred then $\delta\left(\zeta_{j}\right)<r$, for otherwise $\alpha \geq\left|\ell_{j}\left(\zeta_{j}\right)\right|^{2 \beta}=$ $1>\alpha$.

Thus if we define, as before, $\epsilon=K n R_{n}^{2} / \lambda$ for a suitable $K>0$, then $\left\{\zeta_{j}\right\}_{1}^{n} \subset\{\delta(\zeta)<r\}$ with probability at least $1-\epsilon$, or in other words

$$
\mathbf{P}_{n}^{\beta}\left(\left\{D_{n} \geq r\right\}\right) \leq \epsilon .
$$

This is proven whenever $\epsilon \gtrsim n R_{n}^{2} e^{-\beta k_{0} n}$. Taking $\epsilon=e^{-\beta k n}$ where $k=k_{0} / 2$, we finish the proof. Q.E.D.

\section{Concluding remarks}

In this section, shall generalize our main results, by allowing for perturbations of the form $u / n$ where $u$ is a suitable function. After that, we will comment on related results and say something about future prospects.

\subsection{Perturbations of real-analytic potentials}

Let us fix a potential $Q$ obeying the conditions (i)-(vi).

Now pick an arbitrary bounded, measurable, real-valued function $u$ and consider the $n$-dependent potential

$$
V_{n}(\zeta)=Q(\zeta)+\frac{1}{n} u(\zeta)
$$

In a "classical" meaning, the potentials $Q$ and $V_{n}$ are indistinguishable; their droplets and equilibrium measures are the same. The difference between them appears on the statistical level, when we introduce the Gibbs measure corresponding to $V_{n}$,

$$
d \mathbf{P}_{n}^{\beta} \propto e^{-\beta H_{n}} d A_{n}, \quad H_{n}:=\sum_{j \neq k}^{n} \log \frac{1}{\left|\zeta_{j}-\zeta_{k}\right|}+n \sum_{j=1}^{n} V_{n}\left(\zeta_{j}\right) .
$$

More precisely, the weakly $n$-dependent term $(u / n)$ affects the distribution of particles near the boundary.

The present more general situation can be treated similarly as before, by redefining the class $\mathscr{W}_{n}$ of weighted polynomials to consist of elements of the form

$$
f=q \cdot e^{-n V_{n} / 2},
$$

where $q$ is a holomorphic polynomial of degree at most $n-1$. Again we consider this as a subspace of $L^{2}$.

To an element $f=q \cdot e^{-n V_{n} / 2} \in \mathscr{W}_{n}$ we associate the weighted polynomial $\tilde{f}=$ $q \cdot e^{-n Q / 2}=f \cdot e^{u / 2}$. Since $u$ is in $L^{\infty}(\mathbb{C})$ we have

$$
|f(\zeta)| \asymp|\tilde{f}(\zeta)|
$$

uniformly in $f$ and in $\zeta$.

Theorem 4. Under the above hypotheses, Theorem 1 holds up to a constant, i.e., the 1-point function of the ensemble in potential $V_{n}$ satisfies $\mathbf{R}_{n}^{\beta} \lesssim n^{2} e^{s \beta} e^{-c n \beta \delta(\zeta)^{2}}$. Moreover, Theorem 2 and Theorem 3 remain in force as stated (but with $u$-dependent $O$-constants). 
Remark on the proof. In view of (5.1) we have for each measurable subset $W \subset \mathbb{C}$ and each $j, 1 \leq j \leq n$, that $Y_{j, W} \asymp \tilde{Y}_{j, W}$ where

$$
Y_{j, W}=\int_{W}\left|\ell_{j}\right|^{2 \beta} d A \quad \text { and } \quad \tilde{Y}_{j, W}=\int_{W}\left|\tilde{\ell}_{j}\right|^{2 \beta} d A .
$$

The identity $\mathbf{E}_{n}^{\beta}\left(\mathbf{1}_{U}\left(\zeta_{j}\right) \cdot Y_{j, W}\right)=|U| \mathbf{P}_{n}^{\beta}\left(\left\{\zeta_{1} \in W\right\}\right)$ holds as before, and likewise $\tilde{\mathbf{E}}_{n}^{\beta}\left(\mathbf{1}_{U}\left(\zeta_{j}\right)\right.$. $\left.\tilde{Y}_{j, W}\right)=|U| \cdot \tilde{\mathbf{P}}_{n}^{\beta}\left(\left\{\zeta_{1} \in W\right\}\right)$, where tildes are used to indicate the unperturbed distribution (with respect to potential $Q$ ).

Using this, it is easily seen that we lose at most a constant factor when adding the perturbation $u / n$. In Theorem 2 the logarithm of that factor can be absorbed in the constant $\mu$, and likewise our argument for Theorem 3 goes through essentially unaltered.

Remark 5.1. The assumptions (iv) and (vi) on the underlying potential $Q$ are automatic from the others if we assume that $Q$ is real-analytic where it is finite. This follows from Sakai's regularity theorem, see [7]. The perturbation $u / n$ may however be smooth.

Remark 5.2. It is interesting to view the above result from the perspective of the selfimproving method from the paper [4]. This method was developed with a partial intention to eventually obtain a rigorous proof of full plane Gaussian field convergence of linear statistics of a Coulomb gas, but due to some technical challenges it was only applied when $\beta=1$. (The influential paper [21] provides a somewhat analogous construction on $\mathbb{R}$, which was also applied to $\beta$-ensembles.)

One of the technical obstacles for extending the proof to cover $\beta$-ensembles involved having a good enough decay of the 1-point function in the exterior of the droplet, which is a case where Theorem 4 could be relevant.

The problem of proving Gaussian field convergence in the planar case was later subject of some attention, when the papers [11, 22] appeared almost simultaneously, proposing different approaches to its solution. This notwithstanding, given a profound statement there are of course always questions of finding alternative explanations. (For the standard Ginibre ensemble, convergence to the Gaussian field was first proved in [24].)

\subsection{Comparison of Theorem 2 to earlier results}

Suppose that $\beta=1, Q$ is radially symmetric, and $S$ is a disc of radius $R$. In this case we have recognized $R+D_{n}$ as, essentially, the spectral radius of a matrix picked randomly from a certain normal matrix ensemble. The distribution of this spectral radius was worked out by Rider [23] for the Ginibre ensemble (the potential $Q=|\zeta|^{2}$ ) and Chafaï and Péché for more general radially symmetric potentials [16].

For a detailed comparison, we introduce the random variables

$$
\omega_{n}=\sqrt{4 n \gamma_{n} c_{0}}\left(D_{n}-\sqrt{\frac{\gamma_{n}}{4 n c_{0}}}\right), \quad\left(c_{0}=\Delta Q(R)\right) .
$$

The results in $[16,23]$ imply that $\omega_{n}$ converges in distribution to the standard Gumbel distribution as $n \rightarrow \infty$, where

$$
\gamma_{n}=\log (n / 2 \pi)-\log \log n+\log \left(R^{2} c_{0}\right) .
$$

(We may recall here that a random variable $X$ is said to have a standard Gumbel distribution if its distribution function is $\mathbb{P}(X \leq t)=\exp (-\exp (-t))$.)

Natural generalizations of these results for ensembles with various types of "boundary confinements" are given in the papers [6, 26]. 
The theorems of Rider and Chafaï-Péché can be said give a kind of two-dimensional analogue to the well-known convergence to the Tracy-Widom distribution for the top eigenvalue in Hermitian random matrix theory [1, 27]. Numerical evidence in the recent paper [14] indicates that some similar kind of law might hold for the $\beta$-Ginibre ensemble. In this connection, it is interesting to recall that a very precise asymptotic for the tail of the Tracy-Widom $\beta$-distribution for certain one-dimensional ensembles was worked out by Dumaz and Virág in the paper [18].

To further compare with our Theorem 2 we observe that as $n \rightarrow \infty$

$$
\sqrt{\frac{\gamma_{n}}{4 n c_{0}}} \sim \frac{1}{2 \sqrt{c_{0}}} \sqrt{\frac{\log n}{n}}
$$

This is of the same order of magnitude $\left(\sqrt{\frac{\log n}{n}}\right)$ as our present bound in Theorem 2 . Incidentally, we see that our value for the constant $A$ of proportionality in (1.13) can be improved by a factor $1 / 2$ in this case.

The low temperature regime when $\beta \asymp \log n$ was studied in [3]. In such a setting, our present results show that the gas is effectively localized to a microscopic neighbourhood of $S$, i.e., to a neighbourhood of the form

$$
\left\{\zeta ; \delta(\zeta) \lesssim n^{-1 / 2}\right\}
$$

This is used in [8] to analyze low temperature Coulomb systems.

Due to limitations of our methods, we do not seem to quite reach up to high temperatures of the magnitude $\beta \asymp 1 / n$ here. This kind of regime is however studied, in a suitably adapted setting, in the recent paper [2].

\section{A Johansson's convergence theorem}

In this appendix, we present relevant details about Johansson's convergence theorem [21, Theorem 2.1] in the planar setting, with varying $\beta$ 's. As we shall see, the arguments in [21] and [20, Appendix A] carry through (with some slight extra care) provided that $\beta$ is not too small.

Given a confining potential $Q$ it is convenient to introduce the kernel

$$
L_{Q}(\zeta, \eta):=\log \frac{1}{|\zeta-\eta|}+\frac{1}{2}(Q(\zeta)+Q(\eta)) .
$$

If $\mu$ is a compactly supported Borel measure on $\mathbb{C}$, the logarithmic $Q$-energy can be written as

$$
I_{Q}[\mu]=\iint L_{Q}(\zeta, \eta) d \mu(\zeta) d \mu(\eta)=\iint_{\mathbb{C}^{2}} \log \frac{1}{|\zeta-\eta|} d \mu(\zeta) d \mu(\eta)+\mu(Q) .
$$

Let $\sigma=\Delta Q \cdot \mathbf{1}_{S} d A$ be the equilibrium measure in external potential $Q$, i.e., the minimizer among compactly supported unit charges of the functional (A.1). The minimum value of the weighted energy

$$
\gamma(Q):=I_{Q}[\sigma]
$$

is called the "modified Robin constant" in external potential $Q$.

It is pertinent to recall that the assumption (1.1) implies that there are constants $C \in \mathbb{R}$ and $k>1$ such that

$$
Q(\zeta)+C \geq k \log \left(1+|\zeta|^{2}\right), \quad \zeta \in \mathbb{C}
$$


To each configuration $\left\{\zeta_{j}\right\}_{1}^{n}$ we associated the empirical measure $\mu_{n}=\frac{1}{n} \sum_{j=1}^{n} \delta_{\zeta_{j}}$. This measure has infinite $Q$-energy, and we use as a substitute the discrete energy

$$
I_{Q}^{\sharp}\left[\mu_{n}\right]:=\frac{1}{n(n-1)} \sum_{j \neq k} L_{Q}\left(\zeta_{j}, \zeta_{k}\right)=\frac{1}{n(n-1)} \sum_{j \neq k} \log \frac{1}{\left|\zeta_{j}-\zeta_{k}\right|}+\mu_{n}(Q) .
$$

This is closely connected to the Hamiltonian $H_{n}$ in (1.2), namely we have

$$
H_{n}=\sum_{j \neq k} L_{Q}\left(\zeta_{j}, \zeta_{k}\right)+\sum_{j=1}^{n} Q\left(\zeta_{j}\right)=n(n-1) I_{Q}^{\sharp}\left[\mu_{n}\right]+\sum_{j=1}^{n} Q\left(\zeta_{j}\right) .
$$

In the following, we consider $\left\{\zeta_{j}\right\}_{1}^{n}$ as a random sample from the Gibbs measure associated with the potential $Q$ and denote by $\mathbf{R}_{n}^{\beta}$ the 1-point function.

The following "Johansson type" theorem, which partly generalizes [21, Theorem 2.1] and [20, Theorem 2.9] to a case of $n$-dependent $\beta$ 's, is the main result of this appendix.

Theorem A.1. Let $\beta=\beta_{n}$ be a sequence such that $n \beta_{n} \rightarrow \infty$ as $n \rightarrow \infty$. Then for any continuous and bounded function $f$ on $\mathbb{C}$ we have

$$
\frac{1}{n} \int_{\mathbb{C}} f \cdot \mathbf{R}_{n}^{\beta_{n}} d A \rightarrow \sigma(f), \quad n \rightarrow \infty .
$$

To prove this theorem, we fix a small $\epsilon>0$ and form the event

$$
A(n, \epsilon)=\left\{I_{Q}^{\sharp}\left(\mu_{n}\right) \leq \gamma(Q)+\epsilon\right\},
$$

where (as always) $\mu_{n}=\sum_{1}^{n} \delta_{\zeta_{j}}$ is picked randomly with respect to $\mathbf{P}_{n}^{\beta_{n}}$.

Lemma A.2. Fix $a \geq 0$. Then there is a positive integer $n_{0}$ depending on $\epsilon$ but not on $a$ such that if $n \geq n_{0}$, then

$$
\mathbf{P}_{n}^{\beta}\left(\left\{\mu_{N} \notin A(n, \epsilon+a)\right\}\right) \leq e^{-\beta a n(n-1) / 2+c n}
$$

where $c>0$ is a constant depending only on $Q$.

Our proof of Lemma A.2 is in two steps.

We start with the following "entropy estimate" for the partition function $Z_{n}:=$ $Z_{n}^{\beta_{n}}(Q)=\int_{\mathbb{C}^{n}} e^{-\beta_{n} H_{n}} d A_{n}$. (The estimate is found for instance in [20, 21]; cf. also [15, Lemma 4.1] for the corresponding statement in $\mathbb{R}^{d}$.)

Lemma A.3. There are constants $C_{1}$ and $C_{2}$ depending only on $Q$ such that for all $n$

$$
\frac{1}{n^{2}} \log Z_{n}^{\beta_{n}} \geq-\beta_{n}\left(1-C_{1} / n\right) \gamma(Q)+C_{2} / n \text {. }
$$

Proof. Write

$$
Z_{n}=\int_{\mathbb{C}^{n}} \exp \left\{-\beta \sum_{j \neq k} L_{Q}\left(\zeta_{j}, \zeta_{k}\right)-\sum_{j=1}^{n}\left(\beta Q\left(\zeta_{j}\right)+\log \varphi\left(\zeta_{j}\right)\right)\right\} \prod_{j=1}^{n} \varphi\left(\zeta_{j}\right) d A\left(\zeta_{j}\right) .
$$

where $\varphi \geq 0$ is any continuous compactly supported function with $\int_{\mathbb{C}} \varphi d A=1$.

By Jensen's inequality,

$$
\begin{aligned}
\log Z_{n} & \geq \int_{\mathbb{C}^{n}}\left\{-\beta \sum_{j \neq k} L_{Q}\left(\zeta_{j}, \zeta_{k}\right)-\sum_{j=1}^{n}(\beta Q+\log \varphi)\left(\zeta_{j}\right)\right\} \prod_{j=1}^{n} \varphi\left(\zeta_{j}\right) d A\left(\zeta_{j}\right) \\
& =-\beta n(n-1) I_{Q}[\varphi]-n \int_{\mathbb{C}}(\beta Q+\log \varphi) \varphi d A
\end{aligned}
$$


with the understanding that $0 \log 0=0$, and where we write $I_{Q}[\varphi]$ in place of $I_{Q}[\varphi d A]$. This leads to

$$
\frac{1}{n^{2}} \log Z_{n} \geq-\beta I_{Q}[\varphi]+\frac{\beta}{n} I_{Q}[\varphi]-\frac{\beta}{n} \int Q \varphi d A-\frac{1}{n} \int \varphi \log \varphi d A,
$$

For small $\delta>0$ we let $\chi(\zeta):=\delta^{-2} \mathbf{1}_{D(0, \delta)}(\zeta)$ and define a function $\varphi_{\delta}$ by the convolution

$$
\varphi_{\delta}(\zeta):=\chi * \sigma(\zeta)=\frac{\sigma(D(\zeta, \delta))}{\delta^{2}},
$$

where $\sigma=\Delta Q \cdot \mathbf{1}_{S} d A$ is the equilibrium measure.

As $\delta \rightarrow 0$ we have that $I_{Q}\left[\varphi_{\delta}\right] \rightarrow I_{Q}[\sigma]=\gamma(Q)$; see [20, pp. 870-871] for a careful proof of this.

Thus setting $\varphi=\varphi_{\delta}$ in (A.5) and letting $\delta \rightarrow 0$ we obtain

$$
\frac{1}{n^{2}} \log Z_{n} \geq-\beta\left(1-n^{-1}\right) \gamma(Q)-\frac{\beta}{n} \int_{S} Q \Delta Q d A-\frac{1}{n} \int_{S} \Delta Q \log \Delta Q d A .
$$

The finiteness of the two integrals appearing here follows from our assumptions on $Q$.

Proof of Lemma A.2. If $\mu_{n} \notin A(n, \epsilon+a)$, then

$$
I_{n}^{\sharp}\left[\mu_{n}\right] \geq \gamma(Q)+\epsilon+a .
$$

We next note that the assumption (A.3) and the elementary inequality $|\zeta-\eta|^{2} \leq(1+$ $\left.|\zeta|^{2}\right)\left(1+|\eta|^{2}\right)$ for all $\zeta, \eta \in \mathbb{C}$ imply $L_{Q}(\zeta, \eta) \geq \frac{c_{1}}{2}(Q(\zeta)+Q(\eta))-c_{2}$, where $c_{1}=1-1 / k>0$ and $c_{2}=C / k$. This gives the inequality

$$
I_{n}^{\sharp}\left[\mu_{n}\right] \geq \frac{c_{1}}{n} \sum_{j=1}^{n} Q\left(\zeta_{j}\right)-c_{2} .
$$

Now fix a (small) constant $\theta, 0<\theta<1$ and take a convex combination of the inequalities (A.6) and (A.7). We obtain that for all $\mu_{n} \notin A(n, \epsilon+a)$,

$$
I_{n}^{\sharp}\left[\mu_{n}\right] \geq(1-\theta)(\gamma(Q)+\epsilon+a)+\theta\left(\frac{c_{1}}{n} \sum_{j=1}^{n} Q\left(\zeta_{j}\right)-c_{2}\right),
$$

which implies (by use of the inequality (A.3)),

$$
\begin{aligned}
H_{n} & \geq n(n-1)(1-\theta)(\gamma(Q)+\epsilon+a) \\
& +k\left((n-1) \theta c_{1}+1\right) \sum_{j=1}^{n} \log \left(1+\left|\zeta_{j}\right|^{2}\right)-n\left(\theta c_{3} n+c_{4}\right) .
\end{aligned}
$$

Consequently,

$$
\begin{gathered}
\int_{\mathbb{C}^{n} \backslash A(n, \epsilon+a)} e^{-\beta H_{n}} d A_{n} \leq e^{-\beta n(n-1)(1-\theta)(\gamma(Q)+\epsilon+a)+\beta n\left(\theta c_{3} n+c_{4}\right)} \\
\times\left[\int_{\mathbb{C}}\left(1+|\zeta|^{2}\right)^{-k \beta\left((n-1) \theta c_{1}+1\right)} d A(\zeta)\right]^{n} .
\end{gathered}
$$

Since $\int_{\mathbb{C}}\left(1+|\zeta|^{2}\right)^{-\alpha} d A(\zeta)=\frac{1}{\alpha-1}$ for $\alpha>1$, the integral in brackets is no larger than 1 when $n$ is large enough, so

$$
\mathbf{P}_{n}^{\beta}\left(\left\{\mu_{N} \notin A(n, \epsilon+a)\right\}\right) \leq \frac{1}{Z_{n}} e^{-\beta n(n-1)(1-\theta)(\gamma(Q)+\epsilon+a)+\beta n\left(\theta c_{3} n+c_{4}\right)}, \quad n \geq n_{0} .
$$


We now observe that Lemma A.3 implies

$$
Z_{n} \geq \exp \left(-n(n-1) \beta(\gamma(Q)+\epsilon)-c_{5} n\right) .
$$

Then for $n \geq n_{0}$,

$$
\mathbf{P}_{n}^{\beta}\left(\left\{\mu_{N} \notin A(n, \epsilon+a)\right\}\right) \leq e^{\beta n(n-1)\left[\theta\left(\gamma(Q)+c_{3}+\epsilon\right)-(1-\theta) a+o(1)\right]+c_{5} n} .
$$

Finally we fix $\theta$ with $0<\theta<1 / 4$ such that $\theta\left(\gamma(Q)+c_{3}+\epsilon\right)<a / 4$. Then for $n \geq n_{0}$

$$
\mathbf{P}_{n}^{\beta}\left(\left\{\mu_{N} \notin A(n, \epsilon+a)\right\}\right) \leq e^{\beta n(n-1)[a / 4-(1-\theta) a]+c_{5} n} \leq e^{-\beta a n(n-1) / 2+c_{5} n} .
$$

Proof of Theorem A.1. It now suffices to recapitulate a standard argument, which can be found (for example) in [21, p. 195] in the linear case.

Fix a small $\epsilon>0$. For each $n \geq n_{0}(\epsilon)$ we pick with large probability a configuration $\left\{\zeta_{j}\right\}_{1}^{n}$ with $\mu_{n} \in A(n, 2 \epsilon)$. Indeed, by Lemma A.2 the probability for the complementary event is no larger than $e^{-\epsilon \beta n(n-1) / 2+c n}$.

The measures $\mu_{n}$ are then "tight" by (A.3), i.e., given any $m>0$ there is an $R>0$ such that $\mu_{n}(\mathbb{C} \backslash D(0, R))<m$ for all $n$. This means that we can extract weakly convergent subsequences (renamed $\mu_{n}$ ) converging weakly to probability measures $\mu^{\epsilon}$ on $\mathbb{C}$, in the sense that $\mu_{n}(f) \rightarrow \mu^{\epsilon}(f)$ for each continuous and bounded function $f$.

Letting $\epsilon=\epsilon_{n} \downarrow 0$ along a suitable sequence, the measures $\mu^{\epsilon_{n}}$ converge weakly to a probability measure $\mu$ with $I_{Q}[\mu] \leq \gamma(Q)$. This implies $\mu=\sigma$ by unicity of the equilibrium measure, see [25, Theorem I.1.3]. If the convergence $\varepsilon_{n} \rightarrow 0$ is sufficiently slow that $\epsilon_{n} \beta_{n} n \rightarrow \infty$ as $n \rightarrow \infty$, this happens with large probability, tending to 1 as $n \rightarrow \infty$.

We have shown that with probability $1+o(1)$, every subsequence of the measures $\mu_{n}$ has a further subsequence converging weakly to $\sigma$, which shows that the full sequence $\mu_{n} \rightarrow \sigma$ weakly.

In particular, the (uniformly bounded) random variables $\mu_{n}(f)$ converge to $\sigma(f)$ in probability as $n \rightarrow \infty$, where $f$ is a continuous and bounded function. Taking expectations we obtain $\mathbf{E}_{n}^{\beta}\left[\mu_{n}(f)\right] \rightarrow \sigma(f)$ as $n \rightarrow \infty$, i.e., $\frac{1}{n} \int_{\mathbb{C}} f \cdot \mathbf{R}_{n}^{\beta_{n}} d A=\mathbf{E}_{n}^{\beta_{n}}\left[\mu_{n}(f)\right] \rightarrow \sigma(f)$ as $n \rightarrow \infty$.

\section{References}

[1] Akemann, G., Baik, J., Di Francesco, P. (Eds.), The Oxford Handbook of Random Matrix Theory, Oxford 2011. MR-2920518

[2] Akemann, G., Byun, S.-S., The High Temperature Crossover for General 2D Coulomb Gases, J. Stat. Phys. 175 (2019), no. 6, 1043-1065. MR-3962973

[3] Ameur, Y., Repulsion in low temperature $\beta$-ensembles, Commun. Math. Phys. 359 (2018), 1079-1089. MR-3784540

[4] Ameur, Y., Hedenmalm, H., Makarov, N., Ward identities and random normal matrices, Ann. Probab. 43 (2015), 1157-1201. MR-3342661

[5] Ameur, Y., Kang, N.-G., Makarov, N., Rescaling Ward identities in the random normal matrix model, Constr. Approx. 50 (2019), 63-127. MR-3975882

[6] Ameur, Y., Kang, N.-G., Seo, S.-M., On boundary confinements for the Coulomb gas, Anal. Math. Phys. 10, paper no. 68 (2020). MR-4169375

[7] Ameur, Y., Kang, N.-G., Makarov, N., Wennman, A., Scaling limits of random normal matrix processes at singular boundary points, J. Funct. Anal. 278 (2020), 108340. MR-4030288

[8] Ameur, Y., Romero, J. L., The planar low temperature Coulomb gas: separation and equidistribution, arXiv:2010.10179.

[9] Balogh, F., Bertola, M., Lee, S.-Y., McLaughlin, K. D., Strong asymptotics of orthogonal polynomials with respect to a measure supported on the plane, Comm. Pure Appl. Math. 68 (2015), 112-172. MR-3280250 
[10] Bauerschmidt, R., Bourgade, P., Nikula, M., Yau, H.-T., Local Density for Two-Dimensional One-Component Plasma, Commun. Math. Phys. 356 (2017), 189-230. MR-3694026

[11] Bauerschmidt, R., Bourgade, P., Nikula, M., Yau, H.-T., The two-dimensional Coulomb plasma: quasi-free approximation and central limit theorem, Adv. Theor. Math. Phys. 23 (2019), 841-1002. MR-4063572

[12] Can, T., Forrester, P.J., Téllez, G., Wiegmann, P., Singular behavior at the edge of Laughlin states. Phys. Rev. B 89 (2014), 235137.

[13] Carroll, T., Marzo, J., Massaneda, X., Ortega-Cerdà, J., Equidistribution and $\beta$-ensembles. Ann. Fac. Sci. Toulouse Math. (6) 27 (2018), 377-387. MR-3831027

[14] Chafaï, D., Ferré, G., Simulating Coulomb and Log-Gases with Hybrid Monte Carlo Algorithms, J. Stat. Phys. 174 (2019), 692-714. MR-3911782

[15] Chafaï, D., Hardy, A., Maïda, M., Concentration for Coulomb gases and Coulomb transport inequalities, J. Funct. Anal. 275 (2018), 1447-1483. MR-3820329

[16] Chafaï, D., Péché, S., A note on the second order universality at the edge of Coulomb gases on the plane, J. Stat. Phys. 156 (2014), 368-383. MR-3215627

[17] Chau, L.-L., Zaboronsky, O., On the Structure of Correlation Functions in the Normal Matrix Model, Commun. Math. Phys. 196 (1998), 203-247. MR-1643533

[18] Dumaz, L., Virág, B., The right tail exponent of the Tracy-Widom $\beta$ distribution, Ann. Inst. Henri Poincaré Probab. Stat. 49 (2013), no. 4, 915-933. MR-3127907

[19] Elbau, P., Felder, G., Density of Eigenvalues of Random Normal Matrices, Commun. Math. Phys. 259 (2005), 433-450. MR-2172690

[20] Hedenmalm, H., Makarov, N., Coulomb gas ensembles and Laplacian growth, Proc. London. Math. Soc. 106 (2013), 859-907. MR-3056295

[21] Johansson, K., On fluctuations of eigenvalues of random Hermitian matrices, Duke Math. J. 91 (1998), 151-204. MR-1487983

[22] Leblé, T., Serfaty, S., Fluctuations of two-dimensional Coulomb gases, Geom. Funct. Anal. 28 (2018), no. 2, 443-508. MR-3788208

[23] Rider, B., A limit theorem at the edge of a non-Hermitian random matrix ensemble, J. Phys. A. 36 (2003), 3401-3409. MR-1986426

[24] Rider, B., Virág, B., The noise in the circular law and the Gaussian free field, Int. Math. Res. Not. IMRN 2007, no. 2, Art. ID rnm006, 33 pp. MR-2361453

[25] Saff, E. B., Totik, V., Logarithmic potentials with external fields, Springer 1997. MR-1485778

[26] Seo, S.-M., Edge scaling limit of the spectral radius for random normal matrix ensembles at hard edge, J. Stat. Phys. 181 (2020), 1473-1489. MR-4179777

[27] Tracy, C. A., Widom, H., Level-spacing distributions and the Airy kernel, Commun. Math. Phys. 159 (1994), 151-174. MR-1257246

[28] Zabrodin, A., Wiegmann, P., Large $N$ expansion for the $2 D$ Dyson gas, J. Phys. A: Math. Gen. 39 (2006), 8933-8964. MR-2240466

Acknowledgments. I am grateful to Seong-Mi Seo and Djalil Chafaï for comments and appreciated help. 


\section{Electronic Journal of Probability Electronic Communications in Probability}

\section{Advantages of publishing in EJP-ECP}

- Very high standards

- Free for authors, free for readers

- Quick publication (no backlog)

- Secure publication $\left(\mathrm{LOCKSS}^{1}\right)$

- Easy interface (EJMS²)

\section{Economical model of EJP-ECP}

- Non profit, sponsored by $\mathrm{IMS}^{3}, \mathrm{BS}^{4}$, ProjectEuclid ${ }^{5}$

- Purely electronic

\section{Help keep the journal free and vigorous}

- Donate to the IMS open access fund ${ }^{6}$ (click here to donate!)

- Submit your best articles to EJP-ECP

- Choose EJP-ECP over for-profit journals

\footnotetext{
${ }^{1}$ LOCKSS: Lots of Copies Keep Stuff Safe http://www. lockss.org/

${ }^{2}$ EJMS: Electronic Journal Management System http://www.vtex.lt/en/ejms.html

${ }^{3}$ IMS: Institute of Mathematical Statistics http://www.imstat.org/

${ }^{4}$ BS: Bernoulli Society http://www. bernoulli-society.org/

${ }^{5}$ Project Euclid: https://projecteuclid.org/

${ }^{6}$ IMS Open Access Fund: http://www.imstat.org/publications/open.htm
} 